\title{
Factor XIII in Human Plasma and Platelets
}

\author{
Jan McDonagh, R. P. McDonagh, Jr., J.-M. Delâge, and \\ ROBERT H. WAGNER \\ From the Departments of Pathology and Physiology, University of North Carolina \\ School of Medicine, Chapel Hill, North Carolina 27514, and Hôpital du \\ St. Sacrement, Quebec, P.Q., Canada
}

A в S T R A C T Plasma and platelet factor XIII levels were measured in normal human donors and in a patient congenitally deficient in factor XIII. The purpose of these experiments was to study the role of platelet factor XIII in blood coagulation. On polyacrylamide disc electrophoresis, factor XIII activity in extracts of washed normal platelets appeared as a single peak. This peak was missing or very low when factor XIIIdeficient platelet extract was used. The patient was also studied before and after transfusion of fresh frozen plasma. Before transfusion, factor XIII activity could not be detected in the patient's plasma or platelet extracts. $24 \mathrm{hr}$ after transfusion the plasma factor XIII level was still at the anticipated value, but factor XIII activity could not be detected in the platelet extracts. These experiments imply that plasma factor XIII was not transported across the platelet membrane in measurable quantities in vivo. This suggests that platelet factor XIII is a true platelet component and originates in the platelet precursor, the megakaryocyte. Thrombelastographic studies suggest that platelet factor XIII participates in the coagulation process. Thrombelastograms of factor XIII-deficient samples had decreased maximum amplitude and clot elasticity values. The abnormalities were fully corrected by the addition of washed normal platelets to factor XIII-deficient plasma ; preincubation of the normal platelets in the deficient plasma had no additional effect. This indicates that platelet factor XIII is immediately available during clot formation.

\section{INTRODUCTION}

Factor XIII (fibrin stabilizing factor, FSF) is the precursor of an enzyme involved in the formation of the normal blood clot and is present both in plasma (1) and in blood platelets $(2-4)$. It is converted to the active enzyme, $\mathrm{FSF}^{*}$, by thrombin in the presence of calcium

Received for publication 4 November 1968 and in revised form 27 December 1968. ions $(5,6)$. Thrombin converts fibrinogen to fibrin monomer by a process of limited proteolysis in which small fibrinopeptides are released from fibrinogen. Fibrin monomers promptly form polymers which aggregate to yield a gel. This gel is called soluble fibrin because it is readily dispersed in a number of solvents such as $1 \%$ monochloroacetic acid and $5 \mathrm{~m}$ urea. In the presence of calcium, $\mathrm{FSF}^{*}$ catalyzes the formation of covalent bonds between the $\epsilon$-amino group of lysines and $\gamma$-carboxyl group of glutamic acids, so that the fibrin molecules are cross-linked in a transpeptidation reaction (7-9). The cross-linked fibrin is insoluble in monochloroacetic acid and urea. When this reaction is completed, a mechanically strong clot results.

Congenital deficiency of factor XIII is characterized by several features which are peculiar to this defect and others which are common to most coagulopathies. The first and most common symptom is bleeding from the umbilicus, usually during the second week of life (10). Other characteristics observed include frequent hematomas and ecchymoses occurring 24-36 hr after mild trauma, delayed bleeding after cuts and tooth extractions, easy and frequent bruising, and poor wound healing. In a few cases, spontaneous hemarthroses (11) and decidual bleeding with spontaneous abortion of pregnancy $(12,13)$ have been observed. Laboratory tests which are known to be abnormal in factor XIII deficiency are clot solubility and the thrombelastogram.

The purpose of this paper is to report some recent observations involving a patient (G. P.) who was first reported to be congenitally deficient in factor XIII by Barry and Delâge (14). Our studies indicate that platelet factor XIII is an original constituent of platelets and is not derived from plasma. Furthermore, platelet factor XIII participates in the coagulation process and is necessary for the formation of the normal thrombus.

\section{METHODS}

Plasmas. All blood, except for samples for thrombelastography, was obtained from human donors by antecubital venipuncture and was collected into Fenwal blood pack bags 
containing $0.15 \mathrm{ml}$ of acid-citrate-dextrose (ACD) formula $\mathrm{A}$, per $\mathrm{ml}$ of blood. Platelet-poor plasma was obtained by double centrifugation at $3500 \mathrm{rpm}$ at $4^{\circ} \mathrm{C}$ for $30 \mathrm{~min}$. This plasma was aspirated with a needle and syringe. Plateletrich plasma was prepared by centrifuging blood at $1000 \mathrm{rpm}$ for $10 \mathrm{~min}$ at $4^{\circ} \mathrm{C}$. Platelet-rich plasma was aspirated with a silicone-coated pipette. In this process, good platelet yield was sacrificed in order to effect quantitative removal of red cells.

Washed platelets. Washed platelets were prepared from freshly drawn blood according to a modification of the procedure of Brinkhous et al. (15). Platelet-rich plasma was adjusted to $\mathrm{pH} 6.5$ with $1.0 \mathrm{M}$ citric acid and centrifuged at $4^{\circ} \mathrm{C}$ to sediment the platelets. Platelets were resuspended in 3-5 ml citrated saline (normal saline containing 1/20 volume trisodium citrate), and the suspension was diluted to $25 \mathrm{ml}$ with citrated saline. The washing process was repeated 8-10 times. No factor XIII activity could be detected in the wash fluid after the fifth or sixth washing. The final suspension of platelets from $500 \mathrm{ml}$ of platelet-rich plasma was in 3-5 $\mathrm{ml}$ citrated saline. With this procedure, the final platelet suspension was of high purity, but the yield was poor. Degree of homogeniety and platelet concentration were determined by phase-contrast microscopy.

Platelet extract. Platelet extracts were prepared by disrupting final platelet suspensions in a Sorvall Omni-mixer with microattachment, which was operated at half maximum speed. After disruption, platelet debris was sedimented by centrifugation, and the supernatant extract was drawn off

Fibrinogen. Human fibrinogen, prepared by $\beta$-alanine precipitation (16), contained $95 \%$ clottable protein. Fibrinogen, which was used as a substrate in the factor XIII assay, was treated with $p$-chloromercuribenzoic acid sodium salt ( $\mathrm{pCMB}$ ) to inactivate trace amounts of factor XIII (17). Unreacted $\mathrm{pCMB}$ was removed by filtration on Sephadex G-25 (Pharmacia Fine Chemicals).

Factor XIII assay. This assay is based on the differential solubility of cross-linked and noncross-linked fibrin in $1 \%$ monochloroacetic acid. For the assay, each test tube contained the following reagents : $1.0 \mathrm{ml}$ of $50 \mathrm{mg} \%$, pCMBtreated fibrinogen; $0.2 \mathrm{ml}$ test material in serial dilutions; $0.1 \mathrm{ml}$ of $0.175 \mathrm{M} \mathrm{CaCl}_{2} ; 0.1 \mathrm{ml}$ thrombin (Topical, Parke, Davis \& Co.), $100 \mathrm{NIH} \mathrm{U/ml.} \mathrm{Diluents} \mathrm{for} \mathrm{the} \mathrm{test} \mathrm{material}$ were citrated saline or citrated saline containing $0.3 \%$ bovine serum albumin (Armour). The reaction mixture was incubated at $37^{\circ} \mathrm{C}$ for $30 \mathrm{~min}$. Then $1.4 \mathrm{ml}$ of a $2 \%$ solution of monochloroacetic acid was added to each tube, and the clots were observed for dissolution. The factor XIII level of the test material was defined as the reciprocal of the highest dilution used in the test system which caused a visible clot to remain $10 \mathrm{~min}$ after the addition of monochloroacetic acid. For controls $0.2 \mathrm{ml}$ diluent was used in place of test material. Control clots always dissolved within $4 \mathrm{~min}$. A constant amount of purified fibrinogen was used in the test system, and fibrinogen present in the test material had no additional effect.

Disc electrophoresis. Analytical disc electrophoresis in $7 \%$ polyacrylamide gel was performed according to the procedure of Davis (18). Equipment and reagents, obtained from Canal Industrial Corporation, Rockville, Md., were used throughout these experiments. Samples were applied as a layer above the stacking gel. The volumes used were 75-100 $\mu \mathrm{l} /$ column. The electrophoresis apparatus was operated at 5 ma for 35-45 min, samples were stained with Amidoschwartz, and electrophoretic patterns were recorded by tracing the gels in a densitometer. After electrophoresis of platelet extract, nonstained gels were tested for factor XIIJ activity. This was done by slicing simultaneously several gels, combining the equivalent sections, and adding 1-2 ml

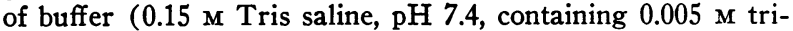
sodium citrate). The gel sections were then disrupted at $4^{\circ} \mathrm{C}$ with a Sorvall Omni-mixer, and proteins were eluted by $12-24 \mathrm{hr}$ standing at $4^{\circ} \mathrm{C}$. Assays were then performed on the eluates. Diluted human fibrinogen preparations were also analyzed.

Thrombelastography. Thrombelastograms were prepared in a thrombelastograph (Hellige, Inc., Garden City, L. I., N. Y.) according to the procedure of Hartert (19). Samples of whole blood, platelet-rich plasma, and platelet-poor plasma were examined. Blood was drawn from normal donors and from the factor XIII-deficient patient into silicone-treated syringes with a two syringe technique. Whole blood, $0.36 \mathrm{ml}$, was put in a stainless steel cuvette, placed in the machine, and the piston was lowered. Platelet-rich and platelet-poor plasmas were prepared from whole blood which had been mixed with one-ninth part of $0.109 \mathrm{M}$ trisodium citrate. Plasma with platelets was obtained after careful differential centrifugation to remove red and white cells. These samples, $0.25 \mathrm{ml}$, were recalcified by addition of $0.10 \mathrm{ml}$ of $0.100 \mathrm{M}$ $\mathrm{CaCl}_{2}$ with careful mixing by raising and lowering piston. The thrombelastogram was begun $30 \mathrm{sec}$ after the addition of calcium. The film speed of the thrombelastograph was 2 $\mathrm{mm} / \mathrm{min}$. The standard notation is used to describe the thrombelastograms : $\mathrm{t}=$ time $(\mathrm{min}), \mathrm{a}=$ amplitude $(\mathrm{mm})$, and $\mathrm{ma}=$ maximum amplitude $(\mathrm{mm})$.

(1) $r$, the reaction time, indicates the beginning of fibrin formation : $r(\mathrm{~min})=($ distance $(\mathrm{mm})$ from $\mathrm{t}=0$ to $\mathrm{a}=1) / 2$ + filling time.

(2) $\mathrm{k}$, the coagulation time, is a measurement of the time required for a clot of a definite solidity to develop : $\mathrm{k}(\mathrm{min})=$ (distance from $a=1$ to $a=20$ ) $/ 2$.

(3) $\epsilon$, the maximum elasticity, represents the elastic properties of the fibrin clot: $\epsilon=(100 \times \mathrm{ma}) /(100-\mathrm{ma})$.

In certain experiments normal platelets were suspended in factor XIII-deficient platelet-poor plasma and the resulting mixture was examined in the thrombelastograph. Normal platelets, washed until no factor XIII activity could be detected in the wash fluid, were concentrated. $0.1 \mathrm{ml}$ of the platelet concentrate was suspended in $1.0 \mathrm{ml}$ of the factor XIII-deficient plasma to give a final concentration of $130,000 / \mathrm{mm}^{8}$. This was the same as the final concentration of platelets measured in the patient's plasma after careful differential centrifugation. In one experiment, normal platelets and factor XIII-deficient plasma were incubated for $1 \mathrm{hr}$ at $37^{\circ} \mathrm{C}$ before beginning the thrombelastogram. In another experiment there was no preincubation, and the platelets were added to the plasma immediately before the mixture was placed in the cuvette. All experiments were run in duplicate.

Factor XIII-deficient patient. G. P. is an alert, normally developed $16 \mathrm{yr}$ old male who weighs $50 \mathrm{~kg}$. His history is typical for congenital deficiency of factor XIII. He suffered from bleeding from the umbilicus which began shortly after birth and lasted for 21 days. He has had multiple hematomas and ecchymoses secondary to trauma. Factor XIII deficiency was diagnosed in this patient in 1965, when he suffered a severe cerebral hemorrhage following minor head trauma (14). He is currently maintained on prophylactic plasma transfusions at 3 -month intervals. 


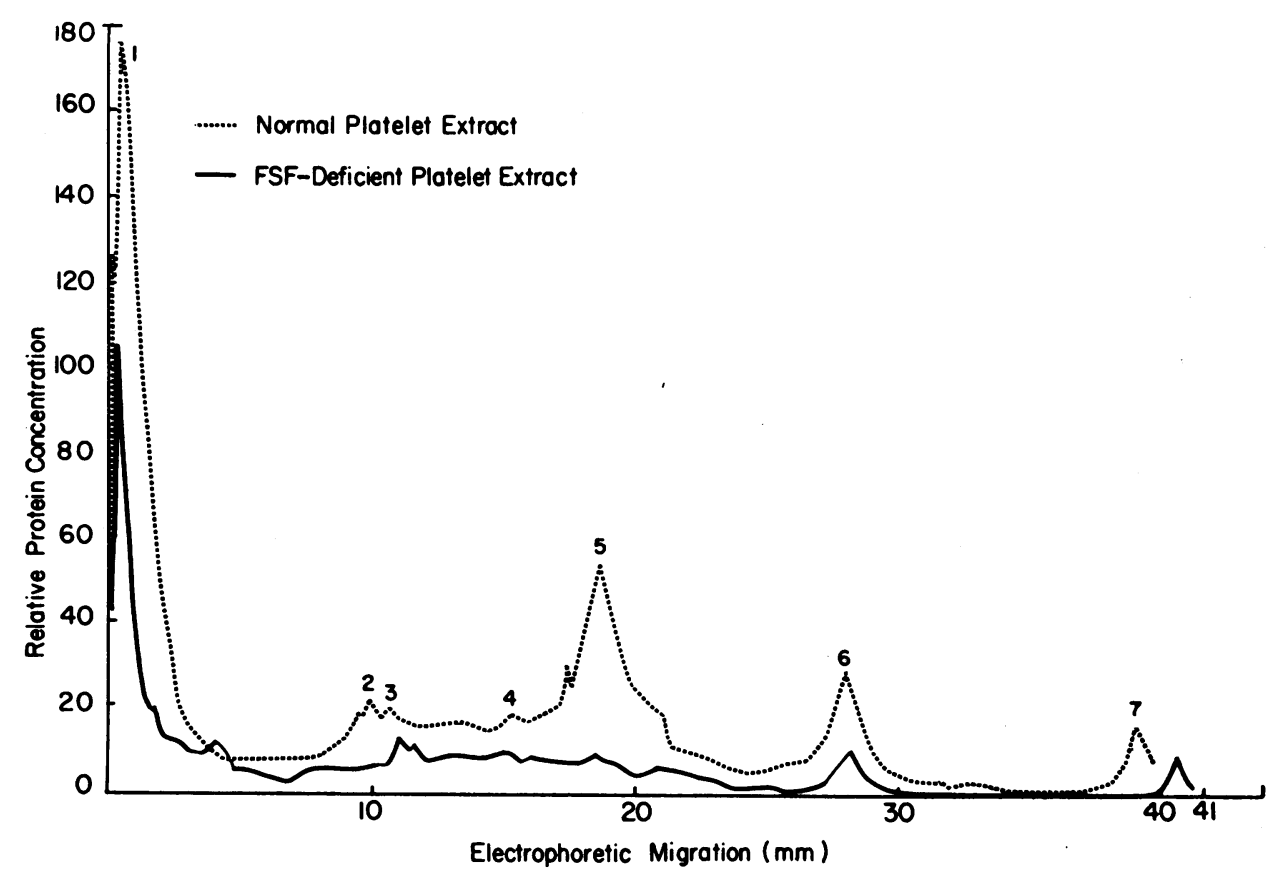

FIGURE 1 Electrophoretic migration of normal and factor XIII-deficient platelet extracts in $7 \%$ polyacrylamide gel at $\mathrm{pH} 9.5$. Arabic numbers designate the major peaks.

Since 1966 G. P. has been studied on four occasions in the Clinical Research Unit of North Carolina Memorial Hospital. At the time of his visits, which were always 2-3 months after his last transfusion, plasma factor XIII levels were zero.

\section{RESULTS}

Polyacrylamide electrophoresis of platelet extracts. Four samples of factor XIII-deficient platelet extract and seven samples of normal platelet extracts were sub- jected to analytical disc electrophoresis in $7 \%$ polyacrylamide gel. Typical results of the electrophoretic migration patterns for both types of samples are shown in Fig. 1. In both samples the extracts were prepared from $3 \mathrm{ml}$ of washed platelets at a concentration of $1 \times 10^{\circ} \%$ $\mathrm{mm}^{8} .100 \mu \mathrm{l}$ of each platelet extract, equivalent to the contents of $1 \times 10^{3}$ platelets, was applied to each gel. The normal sample contained approximately $45 \mu \mathrm{g}$ of total protein and the factor XIII-deficient sample $38 \mu \mathrm{g}$.

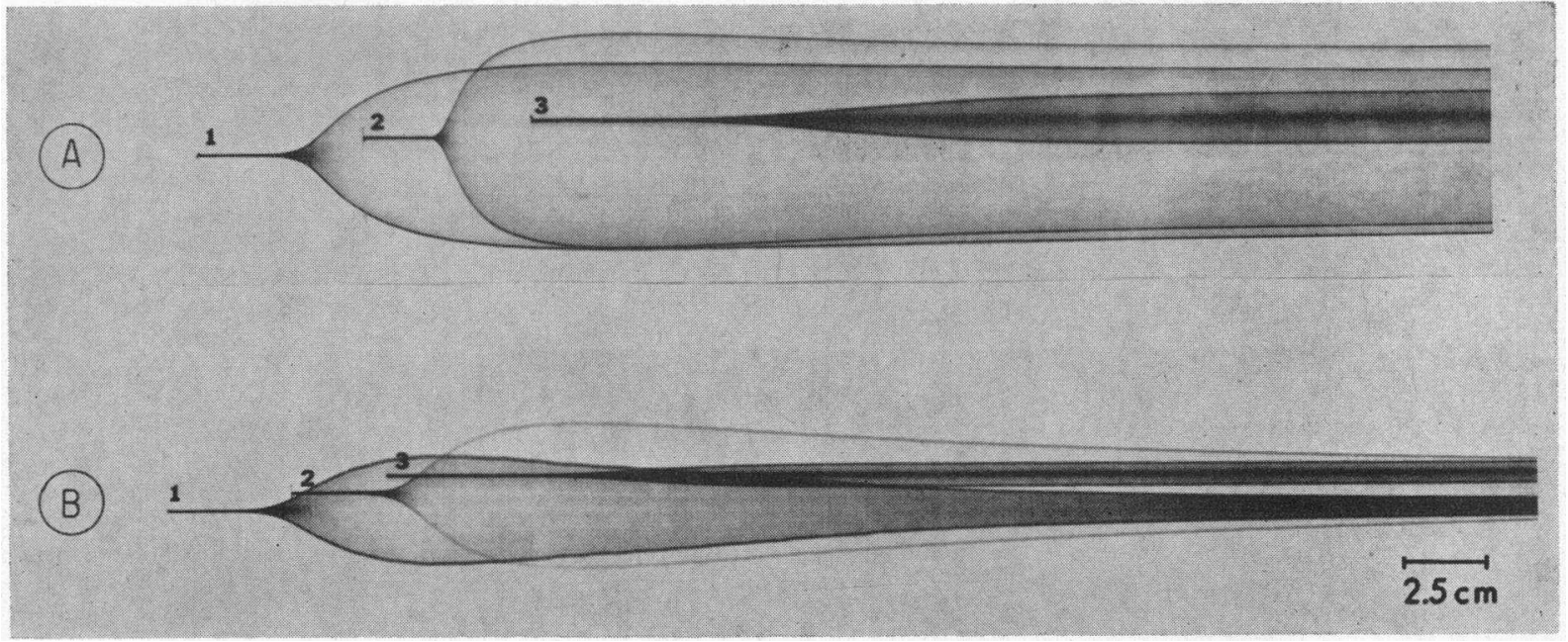

FIGURE 2 Thrombelastograms of (1) whole blood, (2) platelet-rich plasma, and (3) plateletpoor plasma. A : normal samples; B : factor XIII-deficient samples. 
TABLE I

Parameters for Thrombelastograms of Normal and Factor XIII-Deficient Blood

\begin{tabular}{lrrrrr}
\hline & $\begin{array}{c}\text { Platelet } \\
\text { concentration }\end{array}$ & r & k & ma & \multicolumn{1}{c}{} \\
\hline & count $/ \mathrm{mm}^{2}$ & $\min$ & $\min$ & $\mathrm{mm}$ & \\
Normal samples & & & & & \\
Whole blood & 300,000 & 15.7 & 7.6 & 56.5 & 130.0 \\
Platelet-rich plasma & 150,000 & 11.4 & 3.0 & 64.6 & 182.3 \\
Platelet-poor plasma & 300 & 28.5 & $*$ & 15.4 & 18.2 \\
& & & & & \\
Deficient samples & & & & & \\
Whole blood & 295,000 & 15.8 & 11.2 & 32.5 & 48.2 \\
Platelet-rich plasma & 132,000 & 14.1 & 8.2 & 43.5 & 77.0 \\
Platelet-poor plasma & 300 & 28.9 & $*$ & 3.8 & 3.9 \\
\hline
\end{tabular}

* Not measurable.

Some differences in intensity of staining were observed. Factor XIII assays of eluates from sections of the gels containing normal extracts showed that all of the activity was confined to peak 5 . The relative activity of peak 5 was 32 ; for all other fractions it was zero. This is a major peak in electrophoresis of normal platelet extract and is absent or greatly diminished in extracts prepared from the factor XIII-deficient patient. In parallel runs, the migration of human fibrinogen was the same as for peak 1 of the platelet extracts.

Thrombelastography of normal and factor XIII-deficient blood. The thrombelastograms shown in Fig. 2 were obtained with whole blood, platelet-rich plasma, and platelet-poor plasma from a normal donor and from the factor XIII-deficient patient. Data obtained from the thrombelastograms and the platelet concentrations of the various samples are given in Table I. Other thrombelastographic experiments were performed to determine the effect of normal platelets on the thrombelastogram of factor XIII-deficient plasma. Typical tracings for these experiments are shown in Fig. 3. These include
TABLE II

Parameters for Thrombelastograms of Platelet-Rich Plasma Containing Normal Platelets Suspended in Factor XIII-Deficient Piasma

\begin{tabular}{lcc}
\hline Samples & ma & $\epsilon$ \\
\hline & $m m$ & \\
Normal plasma + normal platelets & 59.7 & 146.0 \\
$\begin{array}{l}\text { Deficient plasma + normal platelets, } \\
\text { preincubated for 60 min }\end{array}$ & 65.1 & 186.5 \\
$\begin{array}{l}\text { Deficient plasma + normal platelets, } \\
\text { no preincubation }\end{array}$ & 61.7 & 160.0 \\
\hline
\end{tabular}

(1) the normal control, (2) normal platelets and deficient plasma preincubated for one hour, and (3) normal platelets and deficient plasma with no preincubation. The maximum amplitude and elasticity values which were obtained from the tracings are given in Table II.

Measurement of plasma and platelet factor XIII activity. On his last visit to this research unit, G.P. underwent surgery for the extraction of 13 teeth. Before surgery he received $750 \mathrm{ml}$ of fresh frozen plasma. The hematocrit, fibrinogen concentration, and platelet count of the patient were normal before transfusion. Plasma and platelet factor XIII levels of the deficient patient before and after the plasma infusion are shown in Table III. The range for normal values is also given. At 24 hr post transfusion, the plasma factor XIII activity of the patient was 16; while platelet extract, prepared from washed platelets at a concentration of $7.5 \times 10^{\circ} / \mathrm{mm}^{3}$, had no factor XIII activity.

The patient was not bleeding before transfusion but was transfused prophylactically before surgery. During surgery he lost $300-400 \mathrm{ml}$ of blood. A slight amount of blood oozing from the gums was observed for 2 days after the operation. The plasma factor XIII level of the patient was measured at $1,6,12$, and $24 \mathrm{hr}$ after trans-

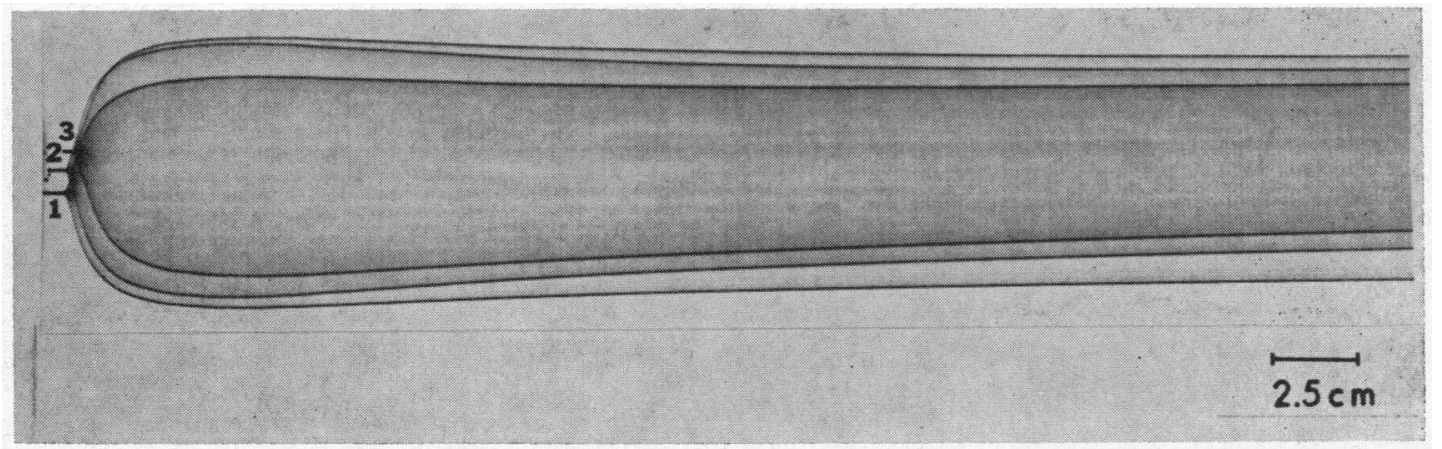

FigURE 3 Thrombelastograms obtained by suspending normal platelets in (1) normal plateletpoor plasma (control), (2) factor XIII-deficient platelet-poor plasma and preincubated for 1 hr at $37^{\circ} \mathrm{C}$, and (3) same as (2) with no preincubation. 
TABLE III

Effects of Transfusion of a Factor XIII-Deficient Patient with Normal Plasma

\begin{tabular}{lcc}
\hline \multicolumn{1}{c}{ Subject } & $\begin{array}{c}\text { Plasma } \\
\text { factor XIII }\end{array}$ & $\begin{array}{c}\text { Platelet } \\
\text { factor XIII }\end{array}$ \\
\hline Normal (20 donors) & $64-128$ & $800-1600$ \\
Patient before transfusion & 0 & $0^{*}$ \\
Patient 24 hr after transfusion & 16 & $0 \ddagger$ \\
of 5 units of fresh frozen plasma & & \\
\hline
\end{tabular}

* Measured in extract of washed platelets at $8 \times 10^{6} / \mathrm{mm}^{3}$.

$\ddagger$ Measured in extract of washed platelets at $7.5 \times 10^{6} / \mathrm{mm}^{3}$.

fusion and thereafter at $24 \mathrm{hr}$ intervals for 6 days. $\mathrm{W}$ ithin the limits of the assay, it was found to remain constant over the 6 day period. This indicates an in vivo half-life greater than 6 days, which is within the range previously reported $(12,20)$.

The patient's mother was also tested for factor XIII activity. The plasma factor XIII level was found to be 128 ; and the platelet factor XIII level, from washed platelets at $14 \times 10^{8} / \mathrm{mm}^{3}$, was 800 .

\section{DISCUSSION}

On polyacrylamide disc electrophoresis of normal platelet extract, factor XIII activity is found in a major peak (Fig. 1, peak 5). This peak is missing when factor XIII-deficient platelet extract is electrophoresed. Depending upon the specific activity of platelet factor XIII, which is unknown, $10 \%$ or more of peak 5 could be platelet factor XIII. It is also interesting that the electrophoretic mobilities of plasma fibrinogen and platelet factor XIII in polyacrylamide were found to be considerably different. Both fibrinogen and peak 1 were found in the stacking gel and were thus excluded from the separating gel layer. Since polyacrylamide electrophoresis separates molecules on the basis of molecular size and charge (21), platelet factor XIII is a smaller molecule than fibrinogen or it has a greater charge, or both. Our earlier studies using Sephadex G-200, which indicated that platelet factor XIII is a smaller molecule than fibrinogen or plasma factor XIII, are in agreement with this $(22,23)$.

The thrombelastograms of this factor XIII-deficient patient agree with those reported for other patients congenitally deficient in factor XIII $(24,25)$. The $r$ values are nearly identical for normal and for factor XIIIdeficient samples (Table I): This would be expected because the patient has no abnormality in the early stages of the clotting reaction and thrombin generation occurs normally. The expected abnormalities in ma and $\epsilon$ values are clearly evident for the deficient samples. The low ma values indicate that a clot of maximum solidity did not form. The low $\epsilon$ values indicate a reduced clot elasticity. In each thrombelastogram of samples from the patient the amplitude, and hence elasticity, declined much more rapidly than in the corresponding normal samples. Decline in amplitude in the thrombelastograms of fibrin clots has been ascribed to fibrinolysis $(26,27)$.

In considering a coagulation factor which is present both in plasma and in platelets, the question arises as to the source of the platelet factor. It may be that the factor is adsorbed from plasma onto the surface of the platelet membrane or transported across the membrane in some manner. The other possibility is that the factor is a true platelet component and originates in megakaryocytes. It is Loewy's opinion that plasma factor XIII is derived from platelets (28). On the other hand, Duckert has reported that platelets free of factor XIII take up activity from normal plasma in vitro, but he did not quantitate the amount (29).

The results reported here indicate that factor XIII was not transferred into the platelet from plasma in a measurable amount in vivo. The factor XIII activity in the plasma and platelets of the factor XIII-deficient patient was measured $24 \mathrm{hr}$ after he was transfused with fresh frozen plasma. If plasma factor XIII were the source of platelet factor XIII, one would expect to find activity in platelets $24 \mathrm{hr}$ after in vivo circulation in the presence of plasma factor XIII. The post-transfusion plasma factor XIII level was 16 , which was within the expected range. However, platelet extract, prepared from washed platelets at $7.5 \times 10^{8} / \mathrm{mm}^{3}$, had no factor XIII activity. That no activity was found in the platelets in this experiment suggests that plasma factor XIII is not the source of platelet factor XIII.

Another question which arises is the function of platelet factor XIII. As yet no specific role has been assigned, and it seems that the circulating level of plasma factor XIII should be sufficient for clot stabilization. However, it has been shown that while a small volume of normal plasma added to the deficient plasma will cause the formation of insoluble fibrin, even an equal volume of normal plasma added to factor XIII-deficient plateletrich plasma will not give a normal thrombelastogram (25).

In contrast, it was shown (Fig. 3. Table II) that normal washed platelets, added in physiologic amounts to factor XIII-deficient plasma, gave normal values for maximum amplitude and clot elasticity in the thrombelastogram. This suggests that a possible function of platelet factor XIII is to provide maximum elasticity to the fibrin clot. In the quantity of platelets used, preincubation of normal platelets in the deficient plasma had no additional effect, since sample 2 and sample 3 (Fig. 3) were both corrected to normal. The lack of an incubation effect shows the ready availability of factor XIII in clot formation. 
It should also be noted that the addition of normal platelets to deficient plasma (Fig. 3) prevents the increased rate of fibrinolysis indicated in the thrombelastograms of the factor XIII-deficient patient (Fig. 2 B). This observation is in agreement with our demonstration that the inhibition of fibrinolysis by factor XIII-deficient platelet extract was significantly less than by normal platelet extract (23).

Platelets disrupted by an improved method (23) have a higher titer for factor XIII activity than found previously. From Table III it is apparent that in a normal person, $30-50 \%$ of the total factor XIII activity in blood is present in the platelets. Because of this high platelet factor XIII activity and because of the evidence from thrombelastograph studies, it would appear that platelet-rich plasma might be preferable to plasma for transfusion therapy in factor XIII deficiency. However, platelets are antigenic and are unsuitable for repeated transfusions $(11,30)$. In vivo tests of the efficacy of platelet transfusion in factor XIII deficiency should therefore be reserved for an episode of catastrophic hemorrhage.

As stated earlier, the only tests known to be abnormal in factor XIII deficiency are the clot solubility test and the thrombelastogram. These, together with clinical history, have been used to establish a diagnosis of factor XIII deficiency. However, it is important to understand that the two tests do not measure the same quantity. The clot solubility test measures the minimum amount of factor XIII required for the formation of a fibrin clot which will not dissolve completely under standard conditions. This test cannot measure the amount of factor XIII necessary for the formation of a fibrin clot which has optimal cross-linkage. On the other hand the thrombelastogram appears to be sensitive to both the amount and the source of factor XIII required for normal clot formation. It is likely that there is a large difference between the factor XIII required to promote the hemostasis in the deficient patient and the factor XIII needed in the thrombelastograph for formation of normal clots.

\section{ACKNOWLEDGMENTS}

Grateful acknowledgment is made to Mrs. Jacque Winslow for her expert technical assistance.

This investigation was supported by Research Grants HE-01648, HE-06350, and HE-5652 from the National Institutes of Health. Patient care was provided by Public Health Research Grant FR-46 to the Clinical Research Unit, University of North Carolina. Dr. Wagner was supported by Public Health Service Research Career Program Award K3-HE-1147.

\section{REFERENCES}

1. Lorand, L. 1950. Fibrin clots. Nature (London). 166: 694.
2. Buluk, K. 1955. An unknown function of blood platelets. Polski Tygod. Lekar. 10: 191.

3. Kiesselbach, T. H., and R. H. Wagner. 1966. Fibrinstabilizing factor: a thrombin-labile platelet protein. Amer. J. Physiol. 211: 1472.

4. Lüscher, E. F. 1957. Ein fibrinstabilisierender Faktor aus Thrombocyten. Schweiz. Med. Wochenschr. 87: 1220.

5. Buluk, K., T. Januszko, and J. Olbromski. 1961. Conversion of fibrin to desmofibrin. Nature (London). 191: 1093.

6. Lorand, L., and K. Konishi. 1964. Activation of fibrin stabilizing factor of plasma by thrombin. Arch. Biochem. Biophys. 105: 58.

7. Pisano, J. J., J. S. Finlayson, and M. P. Peyton. 1968. Cross-link in fibrin polymerized by factor XIII: $\epsilon-(\gamma-$ glutamyl) lysine. Science. 160: 892.

8. Matăcić, S., and A. G. Loewy. 1968. The identification of isopeptide cross-links in insoluble fibrin. Biochem. Biophys. Res. Commun. 30: 356.

9. Lorand, L., J. Downey, T. Gotoh, A. Jacobsen, and S. Tokura. 1968. The transpeptidase system which crosslinks fibrin by $\boldsymbol{\gamma}$-glutamyl- $\epsilon$-lysine bonds. Biochem. Biophys. Res. Commun. 31: 222.

10. Duckert, F., and P. Sigg. 1963. The fibrin stabilizing factor (factor XIII). Proc. Congr. Eur. Soc. Haematol. 1250.

11. Britten, A. F. H. 1967. Congenital deficiency of factor XIII (fibrin stabilizing factor). Amer. J. Med. 43: 751

12. Ikkala, E., G. Myllylä, and H. R. Nevanlinna. 1964. Transfusion therapy in factor XIII (F. S. F.) deficiency. Scand. J. Haematol. 1: 308.

13. Fisher, S. 1965. Fibrinase deficiency. Israel J. Med. Sci. 1: 855 .

14. Barry, A., and J.-M. Delâge. 1965. Congenital deficiency of fibrin-stabilizing factor. N. Engl. J. Med. 272: 943.

15. Brinkhous, K. M., E. C. LeRoy, W. P. Cornell, R. C. Brown, J. L. Hazlehurst, and G. P. Vennart. 1958. Macroscopic studies of platelet agglutination: Nature of the thrombocyte agglutinating activity of plasma. Proc. Soc. Exp. Biol. Med. 98: 379.

16. Straughn, W. R., III, and R. H. Wagner. 1966. A simple method for preparing fibrinogen. Thromb. Diath. Haemorrh. 16: 198.

17. Swigert, S., J. L. Koppel, and J. H. Olwin. 1963. Selective inactivation of fibrin stabilizing factor contaminant in fibrinogen. Nature (London). 198: 797.

18. Davis, B. J. 1964. Disc electrophoresis. II. Method and application to human serum proteins. Ann. N. Y. Acad. Sci. 121: 404.

19. Hartert, H. 1951. Die Thrombelastographie. Eine Methode zur physikalischen Analyse des Blutgerinnungsvorganges. Z. Gesamte. Exp. Med. 117: 189.

20. Losowsky, M. S., R. Hall, and W. Goldie. 1965. Congenital deficiency of fibrin-stabilizing factor: a report of three unrelated cases. Lancet. 2: 156.

21. Ornstein, L. 1964. Disc electrophoresis. I. Background and theory. Ann. N. Y. Acad. Sci. 121: 321.

22. Kiesselbach, T. H. and R. H. Wagner. 1967. Platelet and plasma factor XIII (fibrin stabilizing factor). Thromb. Diath. Haemorrh. Suppl. 26: 155.

23. McDonagh, J., T. H. Kiesselbach, and R. H. Wagner. 1969. Factor XIII and antiplasmin activity in human platelets. Amer. J. Physiol. In press.

24. Duckert, F., E. Jung, and D. H. Shmerling. 1961. A 
hitherto undescribed congenital haemorrhagic diathesis probably due to fibrin stabilizing factor deficiency. Thromb. Diath. Haemorrh. 5: 179.

25. Soulier, J. P., O. Prou-Wartelle, and F. Josso. 1964. Haemorrhagic diathesis with a congenital lack of FSF (factor XIII). Thromb. Diath. Haemorrh. Suppl. 13: 123.

26. von Kaulla, K. N., and R. L. Schultz. 1958. Methods for the evaluation of human fibrinolysis: studies with two combined techniques. Amer. J. Clin. Pathol. 29: 104.

27. Egeblad, K., and T. Astrup. 1967. Effects of human plasma on thrombelastographic patterns produced by fibrinolytic agents in fibrin clots. Brit. J. Haematol. 13: 10.

28. Loewy, A. G. 1968. Discussion to Wirkungsmechanismus des Faktors XIII. Thromb. Diath. Haemorrh. Suppl. 28: 27.

29. Duckert, F. 1968. Discussion to Wirkungsmechanismus des Faktors XIII. Thromb. Diath. Haemorrh. Suppl. 28: 28.

30. Bucher, U. and H. Kummer. 1968. Die praktische Durchführung der Blutplättchentransfusion. Schweiz. Med. Wochenschr. 98: 1635. 